\title{
QUALITY OF PERCEPTION TO QUALITY OF SERVICE MAPPING USING A DYNAMICALLY RECONFIGURABLE COMMUNICATION SYSTEM
}

\author{
G.Ghinea, J.P. Thomas and R.S. Fish \\ Department of Computer Science \\ University of Reading \\ P.O.Box 225, Whiteknights \\ Reading, RG6 6AY, \\ United Kingdom
}

\begin{abstract}
We present an innovative approach for providing en-to-end Quality of Service (QoS) guarantees in a distributed multimedia setting. Quality of Perception (QoP) is a term which encompasses not only a user's satisfaction with the quality of multimedia presentations, but also his/her ability to analyse, synthesise and assimilate the informational content of multimedia displays. The basics of a mapping linking QoP to QoS are then presented and the case for including it in an adaptable protocol is made. A proof of concept implementation based on the Dynamically Reconfigurable Protocol Stacks (DRoPS) project show that such applications can be used to improve QoP, especially in the case of dynamic and complex sequences.
\end{abstract}

\section{Introduction}

Traditional approaches of providing Quality of Service (QoS) to multimedia applications have focused on ways and modalities of ensuring and managing different technical parameters such as delay, jitter and packet loss over unreliable networks. To a multimedia user, however, these parameters have little immediate meaning or impact. Although (s)he might be slightly annoyed at the lack of synchronisation between audio and video streams, it is highly unlikely that (s)he will notice, for instance, the loss of a video frame out of the 25 which could be transmitted during a second of footage, especially if the multimedia video in question is one in which the difference between successive frames is small.

Instead, what the end user is more interested in is that (s)he enjoys the overall multimedia display while at the same time assimilating its informational content, and interesting work has been done as far as appreciating a user's satisfaction with multimedia applications presented with varying QoS levels. This has mainly involved either studying the impact of varying clip frame rates on user's enjoyment of multimedia applications [1], [3], or the bounds within which lip synchronisation can fluctuate without undue annoyance on the viewer's part [10].

The focus of our research has been the enhancement of the traditional view of QoS with a user-level defined Quality of Perception (QoP). This is a measure which encompasses not only a user's satisfaction with multimedia clips, but also his/her ability to perceive, synthesise and analyse the informational content of such presentations. As such, we have investigated the interaction between QoP and QoS and its implications from both a user perspective as well as from a networking angle.

Due to varying network conditions it may not be possible to fully satisfy a user's required QoP (and by implication QoS). This calls for protocols that will, given varying network conditions, adapt to provide a "best effort" QoS (and by implication QoP). Section 2 presents empirical results in the measurement of human QoP given a multimedia presentation. An approach to mapping QoP to QoS is described in Section 3. Section 4 introduces DRoPS. Finally, in Section 5, initial results of a comparative study of the performance of 3 different protocol stacks in the delivery of QoP is presented.

\section{Quality of Perception}

Little is known about the effectiveness of multimedia presentations, whether for education, entertainment or any other activity, on the human user. Each new generation of multimedia systems is driven by technological advances rather than by the impact of new technologies on the user. Current metrics for evaluating the quality of multimedia presentations only focus on the satisfaction side of such 
applications, totally neglecting the duality of multimedia as infotainment.

Although QoS itself gives some (and by no means full) indication of the perceptual content of the presentation, it gives no indication of the value of the presentation in terms of the assimilation and understanding of the informational content of the presentation. We have tried to rectify this state of affairs and have conducted experiments to determine the effectiveness of the presentation to the user from a perceptual viewpoint as well as from a perspective of understanding and assimilation of information - Quality of Perception, for short.

Participants in the experiments were asked to view a set of 12 MPEG-1 video clips of roughly half a minute long each. They had no indication whatsoever of the technical parameters with which the tests were actually run. After each clip had been seen, the users were asked a series of questions (ranging between 10 - 12) about it and their responses duly noted. Their satisfaction with the quality of the presentation was also polled.

QoS parameters which were varied include frame rate $(5$, 15 and 25 frames per second) and colour depth (8 and 24bit). Because of the relative importance of the audio stream in a multimedia presentation [7] as well as the fact that it takes up an extremely low amount of bandwidth (the main resource in networked applications) compared to the video it was decided to transmit audio at full quality during the experiments. A total of 12 users have been tested for each (frame_rate, colour_depth) pair. In summary the results (the reader is referred to [5] for a more detailed coverage) obtained in the QoP experiments show that:

- A significant loss of frames (that is, reducing the frame rate) does not proportionally reduce the user's understanding and perception of the presentation. In fact, in some instances (s)he seemed to assimilate more information, thereby resulting in more correct answers to questions. This is because the user has more time to view a frame before the frame changes (at $25 \mathrm{fps}$, a frame is visible for only $0.04 \mathrm{sec}$, whereas at $5 \mathrm{fps}$ a frame is visible for $0.2 \mathrm{sec}$ ), hence absorbing more information. This observation has implications on resource allocation.

- User assimilation of the informational content of clips is characterised by the wys $<>$ wyg (what you see is not what you get) relation. What this means is that often users, whilst still absorbing information correctly, do not notice obvious cues in the clip. Instead the reasoning process by which they arrive at their conclusions is based a lot on intuition and past experience.

- Users have difficulty in absorbing audio, visual and textual information concurrently. Users tend to focus on one of these media at any one moment, although they may switch between the different media. This implies that critical and important messages in a multimedia presentation should be delivered in only one type of medium, or, if delivered concurrently, should be done so with maximal possible quality.

- The link between perception and understanding is a complex one; when the cause of the annoyance is visible (such as lip synchronisation), users will disregard it and focus on the audio message if that is considered to be contextually important.

- Highly dynamic scenes, although expensive in resources, have a negative impact on user understanding and information assimilation. Questions in this category obtained the least number of correct answers. However the entertainment value of such presentations seem to be consistent, irrespective of the frame rate at which they are shown. The link between entertainment and content understanding is therefore not direct and this is further confirmed by the second observation above.

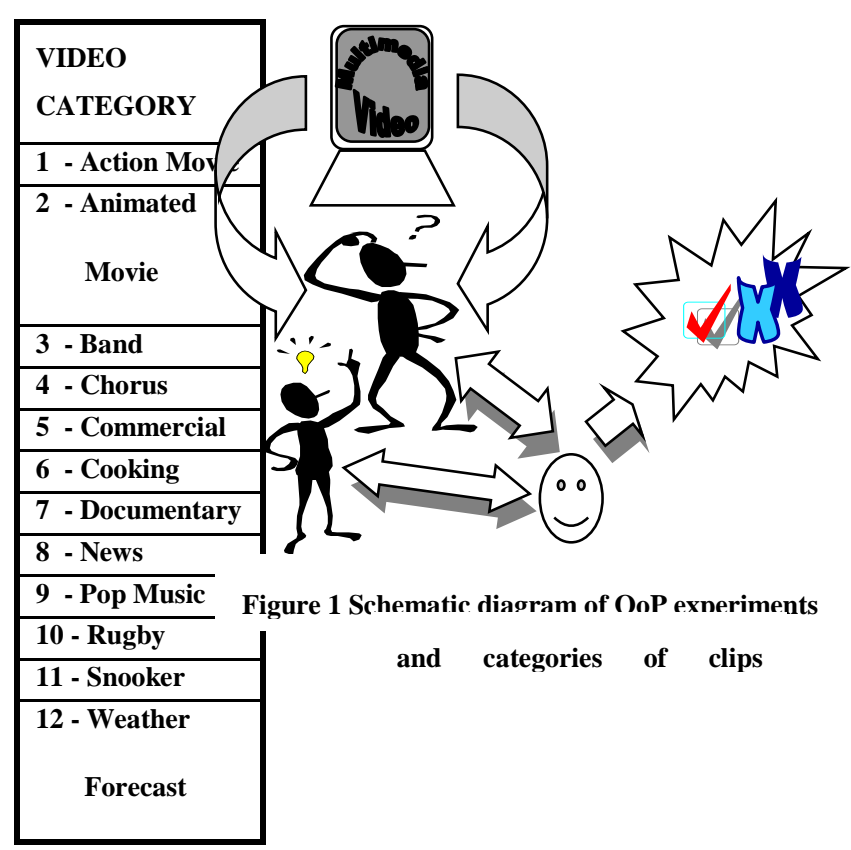


All these results indicate that Quality of Service, typically specified in technical terms such as end-to-end delay, must also be specified in terms of perception, understanding and absorption of content - Quality of Perception - if multimedia presentations are to be truly effective.

\section{QoP to QoS Mapping}

The concept of Quality of Service in distributed multimedia systems is indelibly associated with the provision of an acceptable level of application performance. In turn this performance is itself dependent on both the user-centric Quality of Perception and the robustness of the application to network congestion. Thus, although the problem of multimedia application-level performance can be studied from both a user perspective as well as from a networking angle, it is rarely studied from an integrated viewpoint.

The networking foundation on which current distributed multimedia applications are built either do not specify QoS parameters (also known as best effort transport) or specify them in terms of traffic engineering parameters such as delay, jitter, and loss or error rates. However, these parameters do not convey end-to-end application-specific needs and as a result the underlying network does not consider the sensitivity of the application performance to bandwidth allocation. There is thus an architectural gap between the provision of network-level QoS and application-level QoP requirements of the distributed multimedia applications. This gap causes distributed multimedia systems to inefficiently use network resources and results in poor end-to-end performance. The need for a mapping bridging the gap between application level QoP and network QoS becomes therefore apparent.

In order to achieve this task we have obtained a mapping between the user's high-level QoP and the low-level network parameters. Although a direct mapping cannot be currently obtained, we can specify a relation of proportionality between QoP and QoS. Essentially (please see [4] for full details), the relation is of the form: $Q o P=$ $a_{1} * B E R+a_{2} * S L+a_{3} * S O+a_{4} * D E L+a_{5} * J I T(1)$

Here BER, SL, SO, DEL and JIT represent parameterised QoS values for bit error, segment loss, order, delay and jitter. In [5] it is shown that (1) is in accordance with our QoP results since the coefficients $a_{i}$ include in their expression:
- the dependence of inverse proportionality between a person's QoP and the informational complexity of the clip

- the effect of different media (video, audio or text) as conveyors of information on QoP

- the fact that the mapping is application - specific

In relation (1) we can consider the coefficients $a_{i}$ as describing the relative importance of the low-level network parameters in the context of the application. This leads us to an interesting hypothesis: namely that if one preferentially manages these network QoS parameters (as dictated by their associated weights) then chances are that QoP will be improved.

\section{The Dynamically Reconfigurable Protocol Stacks Project}

One of the ways via which one can preferentially manage network QoS parameters would be an adaptable protocol. Such a protocol is able to actively modify the configuration of a communication system so that at any one time the mechanisms used to transfer data are the most appropriate for the connection over which communication is taking place. Various projects have addressed the protocol issues; XTP [11] allows the exchange of error detection and acknowledgment schemes, Horus [9] the configuration of a fully tailored, but relatively static, communication system and $\mathrm{DaCaPo}$ [8] the maintenance of a tailored protocol configuration.

The DRoPS project provides an infrastructure for the implementation and operation of multiple adaptable protocols. DRoPS based communication systems are composed of fundamental mechanisms called microprotocols [12], that perform arbitrary protocol processing operations. The complexity of processing performed by a microprotocol is not defined by DRoPS and may range from a simple protocol function, such as a checksum, to a complex layer of a protocol stack, such as TCP. A protocol defines header formats, private data structures and an unordered set of microprotocols from which communication systems may be fabricated. Individual protocols are differentiated by these characteristics as well as the semantics of the protocol. Whilst a protocol defines the structure and resources available for constructing a communication system, a 


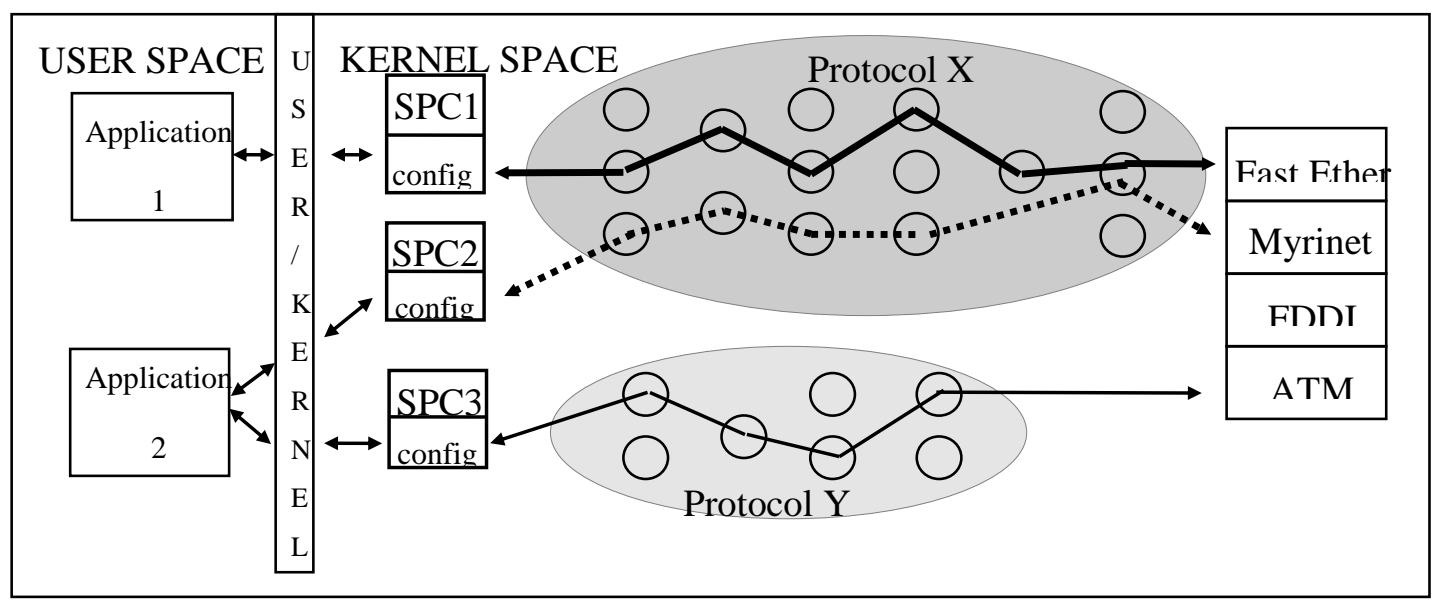

Figure 2 Relation between DRoPS objects, user applications and operating system

protocol stack defines a unique instantiation assigned to a particular connection. In terms of microprotocols, a protocol stack is an ordered set drawn from some parent protocol and combined to form a functional communication system. Each connection is assigned a protocol stack for its sole use, the configuration of which may vary from other stacks derived from the same parent.

DRoPS uses evolutionary techniques (a feed forward multilayer perceptron) for the automation of protocol adaptation [2]. Due to their pattern matching abilities neural networks may be trained to map combinations of required QoS and provided QoS to appropriate protocol configurations. In addition, due to the consistent and continuous nature of this mapping, the neural controller performs generalisation and is able to suggest appropriate protocol configurations for unseen scenarios.

\section{Results}

We are currently in the process of experimenting the suitability of using DRoPS in order to enhance QoP in distributed multimedia environment.

Thus, in the context of a client-server architecture, we have implemented a proof-of-concept application and run tests to evaluate what the effect of DRoPS on QoP is, as opposed to legacy protocol stacks such as TCP/IP and UDP/IP.

Initial results [6] have shown that, although TCP/IP performs remarkably well across a wide variety of multimedia applications, DRoPS can be used to improve QoP, especially in the case of dynamic and complex sequences.
In some instances, of relatively static clips, TCP/IP actually delivers better QoP than DroPS - here the extra processing overheads (a mean of $190 \mu$ s, with a variance of $+/-6 \mu$ s for all cases) incurred by the adaptable protocol are not really warranted. The unreliable data delivery mechanism of UDP/IP has a detrimental across the board impact on QoP

\section{Conclusions and Future Work}

This paper has presented a novel framework for providing end-to-end QoS management in distributed multimedia applications. The main realisation of this objective is through a mapping linking user-centric QoP to network-level QoS, which is then incorporated in an adaptable protocol. Results have shown that there are cases when QoP can thus be improved, especially in the case of dynamic, informationally complex multimedia sequences.

Future work includes refinements of the QoP - QoS mapping and further experiments on evaluating the suitability of using adaptable protocols for providing end-to-end QoS guarantees.

\section{References}

1. R.T. Apteker, J.A. Fisher, V.S. Kisimov, and H. Neishlos, "Video Acceptability and Frame Rate", IEEE Multimedia, 2(3), pp. 32-40, 1995

2. R. S.Fish, R.J.Loader, "Architectural support for runtime adaptable protocols", in Proceedings of the $14^{\text {th }}$ International Conference on Advanced Science 
and Technology (ICAST), Naperville, Illinois, USA, pp. 292-302, 1998

3. K.Fukuda, N. Wakamiya, M. Murata, and H. Miyahara, “QoS Mapping between User's Preference and Bandwidth Control for Video Transport", in Proceedings of the $5^{\text {th }}$ International Workshop on QoS (IWQoS), New York, USA, May 21-23, pp. 291 301, 1997

4. G. Ghinea and J.P. Thomas, "QoS Impact on User Perception and Understanding of Multimedia Video Clips", in Proceedings of ACM Multimedia '98, Bristol, United Kingdom, 1998

5. G. Ghinea and J.P. Thomas, "An Approach Towards Mapping Quality of Perception to QoS in Multimedia Communications", in Proceedings of the IEEE Multimedia Signal Processing Workshop, Helsingor, Denmark, 1999

6. G. Ghinea, J.P. Thomas and R.S. Fish, "Multimedia, Network Protocols and Users - Bridging the Gap", in Proceedings of ACM Multimedia '99, Orlando, Florida, 1999

7. J. Kawalek, "A User Perspective for QoS Management", in Proceedings of the QoS Workshop aligned with the $3^{\text {rd }}$ International Conference on Intelligence in Broadband Services and Network (IS\&N 95), Crete, Greece, 1995

8. T. Plagemann "A Framework for Dynamic Protocol Configuration", PhD Thesis, Swiss Federal Institute of Technology Zurich (Diss. ETH No. 10830), Zurich, Switzerland, 1994

9. R. Renesse, K. Birman, R. Friedman, M. Hayden, D. Karr, "A framework for protocol composition in Horus", in Proceedings of Principles of Distributed Computing, 1995

10. R. Steinmetz, "Human Perception of Jitter and Media Synchronisation", IEEE Journal on Selected Areas in Communications, 14(1), pp. 61 -72, 1996

11. W.T.Strayer, B.J Dempsey, A.C.Weaver, XTP: The Xpress Transfer Protocol, Addison-Wesley, Reading Massachusetts, 1992

12. M. Zitterbart, "A model for flexible and high performance communication subsystems", IEEE Journal on Selected Areas in Communication, 11(1), pp. 507-518, 1993 